\title{
CLUES FROM THE PROMPT EMISSION OF GRB 080319B
}

\author{
Yuan-Chuan Zou ${ }^{1,2}$, Tsvi Piran ${ }^{1}$, And Re'em Sari ${ }^{1,3}$ \\ ${ }^{1}$ The Racah Institute of Physics, Hebrew University, Jerusalem 91904, Israel; tsvi@phys.huji.ac.il \\ ${ }^{2}$ School of Physics, Huazhong University of Science and Technology, Wuhan 430074, China; zouyc@hust.edu.cn \\ ${ }^{3}$ Theoretical Astrophysics, Caltech 130-33, Pasadena, CA 91125, USA; sari@tapir.caltech.edu \\ Received 2008 December 5; accepted 2009 January 6; published 2009 January 28
}

\begin{abstract}
The extremely bright optical flash that accompanied GRB 080319B suggested, at first glance, that the prompt $\gamma$-rays in this burst were produced by synchrotron self-Compton (SSC). We analyze here the observed optical and $\gamma$ spectra. We find that the very strong optical emission imposes, due to self-absorption, very strong constraints on the emission processes and puts the origin of the optical emission at a very large radius, almost inconsistent with internal shock. Alternatively, it requires a very large random Lorentz factor for the electrons. We find that SSC could not have produced the prompt $\gamma$-rays. We also show that the optical emission and the $\gamma$-rays could not have been produced by synchrotron emission from two populations of electrons within the same emitting region. Thus, we must conclude that the optical emission and the $\gamma$-rays were produced in different physical regions. A possible interpretation of the observations is that the $\gamma$-rays arose from internal shocks but the optical flash resulted from external shock emission. This would have been consistent with the few seconds delay observed between the optical and $\gamma$-ray signals.
\end{abstract}

Key words: gamma rays: bursts - radiation mechanism: nonthermal

\section{INTRODUCTION}

The radiation mechanism for the $\gamma$-ray burst (GRB) afterglow is widely accepted as synchrotron emission (see Piran 2004 for a review). However, the mechanism for the prompt emission is still uncertain. Most recently, R. Mochkovitch (2009, in preparation) showed the inconsistency with the overall synchrotron model. On the other hand, Piran et al. (2009) found that synchrotron self-Compton (SSC) cannot explain the prompt emission unless the prompt optical emission is very high. With a naked-eye (5th magnitude) optical flash (Cwiok et al. 2008; Karpov et al. 2008), GRB 080319B (Racusin et al. 2008a) was chosen to be a natural candidate for SSC (Kumar \& Panaitescu 2009).

GRB 080319B was located at redshift $z=0.937$ (Vreeswijk et al. 2008). Its duration $T_{90}$ was $\sim 57 \mathrm{~s}$. The peak flux is $F_{p} \sim 2.26 \pm 0.21 \times 10^{-5} \mathrm{erg} \mathrm{cm}^{-2} \mathrm{~s}^{-1}$ and the peak of the $v F_{v}$ spectrum $E_{p} \simeq 675 \pm 22 \mathrm{keV}$ (i.e., $v_{p} \sim 1.6 \times 10^{20}$ $\mathrm{Hz}$, and consequently $F_{v, p} \sim 1.4 \times 10^{-25} \mathrm{erg} \mathrm{cm}^{-2} \mathrm{~Hz}^{-1} \mathrm{~s}^{-1}$ ). The photon indices below and above $E_{p}$ are $-0.855_{-0.013}^{+0.014}$ and $-3.59_{-0.62}^{+0.32}$, respectively (Racusin et al. 2008b). Choosing standard cosmological parameters $H_{0}=70 \mathrm{~km} \mathrm{~s}^{-1} \mathrm{Mpc}^{-1}, \Omega_{m}=$ $0.3, \Omega_{\lambda}=0.7$, GRB 080319B had a peak luminosity of $L_{p} \sim 9.67 \times 10^{52} \mathrm{erg} \mathrm{s}^{-1}$ and an isotropic equivalent energy of $E_{\gamma, \text { iso }} \simeq 1.32 \times 10^{54} \mathrm{erg}$ (Golenetskii et al. 2008).

The optical observations were going on even before the onset of the GRB because TORTORA was monitoring the same region of sky at that moment. Karpov et al. (2008) reported the optical $V$-band light curve in the prompt phase (from $\sim-10 \mathrm{~s}$ to $\sim 100 \mathrm{~s}$ ). Variability was evident and there were at least three or four pulses in the optical light curve. The peak $V$-band magnitude reached 5.3, corresponding to a flux density of $\sim 28.7 \mathrm{Jy}$.

Models for the optical emission accompanying the prompt $\gamma$-rays have been extensively discussed since the early work of Katz (1994) who used a low-energy spectrum $F_{v} \propto v^{1 / 3}$ and found that the prompt optical emission could be bright to 18th magnitude. The observations of a prompt strong optical flash from GRB 990123 (Akerlof et al. 1999) led to a wave of interest in this phenomenon. The $t^{-2}$ decline of the early optical flash favored a reverse shock model (Sari \& Piran 1999b; Mészáros $\&$ Rees 1999) that was suggested just a few months earlier (Sari \& Piran 1999a). Optical emission from later internal shocks and residual internal shocks have been discussed by Wei et al. (2006) and Li \& Waxman (2008) respectively, and the late internal shock model has been used to interpret the optical flares detected in GRB 990123, GRB 041219a, GRB 050904, and GRB 060111B (Akerlof et al. 1999; Blake et al. 2005; Boër et al. 2006; Klotz et al. 2006; Zou et al. 2006). However, none of the previous bursts had data as plentiful as that of GRB 080319B and the constraints on the models were not very tight.

The very strong optical flash of GRB 080319B leads naturally to the suggestion (Kumar \& Panaitescu 2009; Racusin et al. 2008 b) that the synchrotron of internal shocks produced the prompt optical emission while SSC produced the prompt $\gamma$-rays. We show here that self-absorption of the optical poses major constraints on the source of the optical emission and we consider its implications on general models (including SSC) for the emission of this burst. The Letter is structured as follows: in Section 2, we consider the general constraints that arise from the optical emission. In Section 4, we consider the SSC model and in Section 5 we make general remarks on Inverse Compton (IC). In Section 6, we examine the possibility that the optical and $\gamma$-rays arose from two synchrotron emitting populations of electrons but in the same physical region. We find that the optical and $\gamma$-rays originated in physically different regimes. We discuss the implications of these results in Section 6.

\section{OPTICAL EMISSION}

The very strong optical flash that accompanied GRB 080319B imposes the strongest constraints on the emission mechanism. A lot can be learnt from studying this flash on its own. The observed optical signal, $F_{v, \text { opt }}$, must be less than or equal to the corresponding blackbody emission:

$$
\begin{aligned}
F_{v, \mathrm{opt}} \leqslant F_{\mathrm{BB}} & =2 \pi(1+z)^{3} v_{\mathrm{opt}}^{2} \Gamma \gamma_{e} m_{e}\left(\frac{R}{\Gamma d_{L}}\right)^{2} \\
& =3.5 \times 10^{-24} \gamma_{e, 2} R_{15}^{2} \Gamma_{3}^{-1},
\end{aligned}
$$




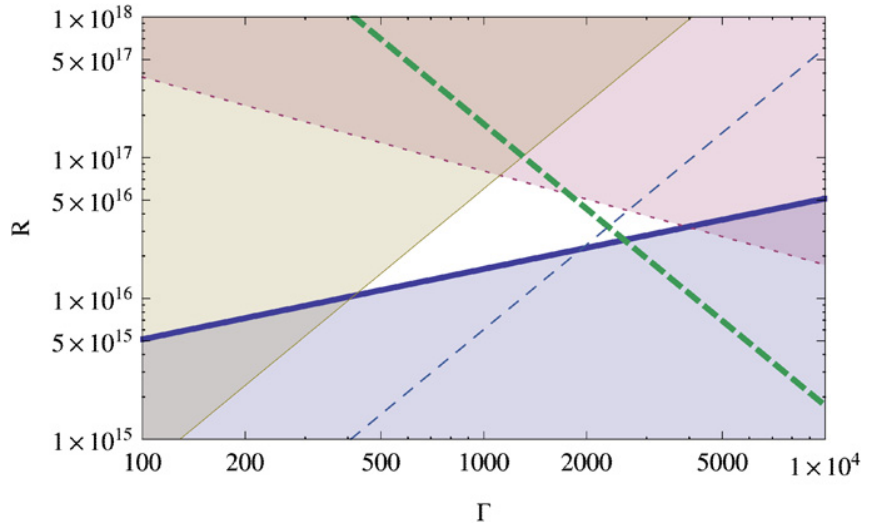

Figure 1. Parameter space in the $(\Gamma, R)$ plane that satisfies the constraints (Equations (1)-(3)). The thick solid line is the blackbody constraint for $\gamma_{e}=100$. The thin solid line indicates the variability timescale (for $\delta t_{\mathrm{opt}}=2$ $\mathrm{s})$. Also shown (thin dashed line) is the variability constraint for $\delta t=0.2 \mathrm{~s}$ corresponding to the $\gamma$-ray variability. The thin dotted line and the thick dashed line are the deceleration radius constraints for ISM and wind environments, respectively. We choose conservatively $E=10^{55} \mathrm{erg}, n=1 \mathrm{~cm}^{-3}$, and $A=3 \times 10^{33} \mathrm{~cm}^{-1}$. The allowed region for ISM with $\gamma_{e}=100$ is the white triangle at the center. It increases slightly for a wind environment.

where $m_{e}$ and $q_{e}$ (that we use later) are the electron rest mass and charge, respectively, $v_{\mathrm{opt}} \sim 5 \times 10^{15} \mathrm{~Hz}$ is the observed optical frequency, $\Gamma$ is the bulk Lorentz factor, $\gamma_{e}$ is the typical electron Lorentz factor $\left(\gamma_{e} m_{e} c^{2} \approx k T\right), R$ is the emission radius, and $d_{L}$ is the luminosity distance. ${ }^{4}$ This value should be compared with the observed optical flux $F_{v, \text { opt }} \sim 2.9 \times 10^{-22} \mathrm{erg} \mathrm{cm}^{-2} \mathrm{~Hz}^{-1} \mathrm{~s}^{-1}$ which is more than 2 orders of magnitude larger than that found for $F_{\mathrm{BB}}$ with "typical" values. This is the essence of the problem of finding a reasonable solution for the emission mechanism in GRB 080319B. By itself this constraint imposes a rather large $\gamma_{e}$ for reasonable values of $R$ and $\Gamma$, or alternatively a very large value of $R$. It will be the major constraint over which models that we examine later fail.

This equation can be combined now with two expressions that link $R$ and $\Gamma$ : the angular timescale,

$$
\delta t>(1+z) \frac{R}{2 \Gamma^{2} c},
$$

and the deceleration radius,

$$
R<R_{\gamma}= \begin{cases}\frac{E}{4 \pi A \Gamma^{2} m_{p} c^{2}}, & \text { wind, } \\ \left(\frac{3 E}{4 \pi n \Gamma^{2} m_{p} c^{2}}\right)^{1 / 3}, & \text { ISM }\end{cases}
$$

where $m_{p}$ is the proton mass, $E$ is the energy of the outflow, $n$ is the interstellar medium (ISM) density and $A$ is the wind parameter.

Figure 1 depicts the allowed region in the $(\Gamma, R)$ phase space that satisfies all three constraints for either wind or ISM environments. One can see that the blackbody limit (Equation (1)) pushes the emitting radius to large values. On the other hand the two other constraints limit $R$ to small values. The allowed region is rather small and the radii are typically large and they will not be consistent with those needed for emitting the $\gamma$-rays. Note that the allowed region shrinks to zero if we take $\delta t \leqslant 0.1 \mathrm{~s}$ as implied from the $\gamma$-ray observations.

\footnotetext{
4 We denote by $A_{i}$ the quantity $A / 10^{i}$ in cgs units.
}

As the condition $R<R_{\gamma}$ applies for internal shocks, this result on its own limits strongly the ability of internal shocks to produce this optical flash. The only way out, within internal shocks, is to increase $\gamma_{e}$ to very large values. However, typical internal shocks involve modest relativistic collisions in which such high Lorentz factors are not common (see, however, Kobayashi \& Sari 2001).

\section{SYNCHROTRON SELF-COMPTON}

We consider an SSC model with minimal assumptions. In fact, unlike the previous section, we do not use the inequalities (2) and (3) that depend on the overall model and we consider only the conditions within the emitting regions. The low-energy (including optical) emission is produced by synchrotron and the $\gamma$-rays are the inverse Compton of this synchrotron emission by the same electrons. We assume that the emitting region is homogeneous and it moves radially outward with a relativistic Lorentz factor $\Gamma$ toward us. It contains $N_{e}$ electrons with a typical Lorentz factor $\gamma_{e}$. Sightly generalizing, we allow for a filling factor $f$ which implies that only a fraction $f$ of the electrons are emitting synchrotron while all the electrons are involved in IC. This can happen, for example, if the magnetic field $B$ occupies only a fraction $f$ of the volume. As we see later, this helps but does not yield a satisfactory solution.

We have four observables, $F_{v, \gamma}, F_{v, \text { opt }}, v_{\gamma}$, and $v_{\text {opt }}$, as the fluxes and frequencies at the $\gamma$ and the optical. We explore what are the conditions $\left(N_{e}, B, \Gamma, \gamma_{e}\right.$, and $\left.R\right)$ needed to produce the observations (see Piran et al. 2009 for a related approach). As we assume that the $\gamma$-rays are produced by IC we have

$$
Y v_{L} F_{v, L}=v_{\gamma} F_{v, \gamma}
$$

and

$$
v_{\gamma}=\gamma_{e}^{2} v_{L}
$$

where $F_{v, L}$ and $v_{L}$ are the (unknown) peak flux and the peak frequency of the low-energy component (that is being IC scattered to produce the soft $\gamma), Y=\gamma_{e}^{2} \tau$ is the Compton parameter, and $\tau$ is the optical depth for Thompson scattering. As the spectral shape is preserved by IC we can use the spectral indices below and above the peak $\gamma$-ray frequency, $\sim 0.2$ and $\sim-2.6$, respectively, to relate the optical flux and the flux at $v_{L}$ $\mathrm{as}^{5}$

$$
F_{v, \mathrm{opt}}=F_{v, L}\left(\frac{v_{\mathrm{opt}}}{v_{L}}\right)^{\alpha},
$$

where $\alpha$ is the observed index at the soft $\gamma$ range. $\alpha$ will be either 0.2 or -2.6 depending on whether $v_{L}$ is larger or smaller than $v_{\text {opt }}$ (which we call UV and IR solutions, respectively). The overall spectral distribution is shown in Figure 2.

Combining these three equations we get

$$
\gamma_{e}=\left(\frac{Y F_{\nu, \mathrm{opt}} \nu_{\gamma}^{\alpha}}{F_{\nu, \gamma} \nu_{\mathrm{opt}}^{\alpha}}\right)^{\frac{1}{2(1+\alpha)}}=530\left(\frac{Y}{140}\right)^{\frac{1}{2(1+\alpha)}} .
$$

We have $\gamma_{e} \sim 70 Y^{0.4}$ for $\alpha=0.2$ corresponding to a UV solution $\left(v_{L}>v_{\text {opt }}\right)$ while for the IR solution $\left(v_{L}<v_{\text {opt }}\right)$ $\alpha=-2.6$ and $\gamma_{e} \sim 2500 Y^{-0.3}$.

\footnotetext{
5 Assuming that $v_{L}$ and $v_{\mathrm{opt}}$ are in the same spectral regime. See subsequent discussion.
} 


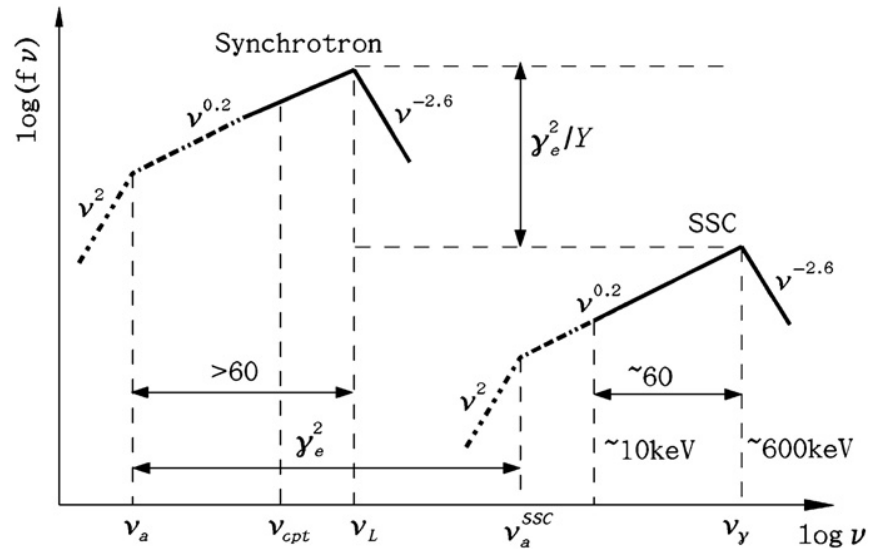

Figure 2. Schematic description of the spectrum in an SSC model. Note that if $10 \mathrm{keV} / \gamma_{e}^{2}>v_{\mathrm{opt}}$, that is if $\gamma_{e}$ is small enough, $v_{\mathrm{opt}}$ might be below $v_{a}$ and in this case Equation (6) should be modified.

Using this expression for $\gamma_{e}$ we now solve the synchrotron frequency and peak synchrotron flux equations:

$$
v_{L}=\frac{1}{(1+z)} \frac{3 q_{e}}{2 \pi m_{e} c} \Gamma B \gamma_{e}^{2}
$$

and

$$
F_{v, L}=\frac{m_{e} c^{2} \sigma_{T}}{3 q_{e}} \frac{(1+z)}{4 \pi d_{L}^{2}} \Gamma B f N_{e} .
$$

Note that $\Gamma$ and $B$ appear as a product and hence we have two equations for two variables, $N_{e}$ and $\Gamma B$, within the emitting region. Once we know $N_{e}$ we can solve for $R$, using the optical depth $\tau=Y / \gamma_{e}^{2}$ and remembering $\tau=\sigma_{T} N_{e} / 4 \pi R^{2}$ :

$$
R=\frac{1}{1+z} \sqrt{\frac{3 d_{L}^{2} q_{e}^{2} F_{\nu, \gamma} \gamma_{e}^{8}}{2 \pi c^{3} f m_{e}^{2} \nu_{\gamma} Y^{2}}} \approx 2 \times 10^{15} \frac{\gamma_{e, 2}^{4}}{\sqrt{f} Y}
$$

Turning now to the flux limit (Equation (1)) we find

$$
\frac{F_{\mathrm{BB}}}{F_{\nu, \mathrm{opt}}}=\frac{3(1+z) q_{e}^{2} F_{v, \gamma} \gamma_{e}^{9} v_{\mathrm{opt}}^{2}}{c^{3} f F_{\nu, \mathrm{opt}} \Gamma m_{e} v_{\gamma} Y^{2}}=0.05 \frac{\gamma_{e, 2}^{9}}{f \Gamma_{3} Y^{2}} .
$$

Substitution of the expression for $\gamma_{e}$ into this equation yields for $\alpha=0.2$ :

$$
\frac{F_{\mathrm{BB}}}{F_{v, \mathrm{opt}}}=2 \times 10^{-3} \frac{Y^{1.75}}{f \Gamma_{3}} .
$$

This ratio is not sensitive to the value of $\alpha$ used. It is clear that for reasonable values of $\Gamma$ this ratio is less than unity unless $Y$ is extremely large. This is the essence of the optical selfabsorption problem that forbids any low $Y$ SSC solution. A large $Y$ will lead to an energy crisis where most of the energy of this (already very powerful) burst would have been emitted in the $\mathrm{GeV}$ regime leading to a huge overall energy requirement. Note that the isotropic equivalent $\gamma$-ray energy of this burst is already larger than $10^{54} \mathrm{erg}$.

Recalling that there is no obvious break in the $\gamma$-ray spectrum from the peak frequency $\sim 600 \mathrm{keV}$ down to $\sim 10 \mathrm{keV}$, a selfabsorption break should not appear in the range $\left[v_{L} / 60, v_{L}\right]$. This sets an even more powerful constraint: $F_{\mathrm{BB}}\left(v_{L} / 60\right)>$ $F_{v_{L} / 60}$ which is typically significantly more difficult to satisfy (by ratio $\left.\left(v_{L} / 60 v_{\mathrm{opt}}\right)^{2-\alpha}\right)$ than Equation (1).
We now briefly consider three caveats for the above result.

1. The filling factor $f$ allows for a more reasonable solution. But one needs an extremely small $f$ for a valid one.

2. In a very small region of the parameter phase space, that is, if $10 \mathrm{keV} / \gamma_{e}^{2}>v_{\text {opt }}$ (see Figure 2), an "optically thick" UV solution is possible. In this case, $F_{v}$ increases like $v^{2}$ from $v_{\text {opt }}$ to $v_{L} / 60$. The solution is slightly different from that given above (as Equation (6) has to be modified) but qualitatively the results remain unchanged (see Figure 2).

3. For the IR solution $(\alpha=-2.6)$, from Equation (7) we find $\gamma_{e} \sim 3 \times 10^{3}$. This leads to a huge emission radius (see Equation (10)) which is larger than $\sim 10^{20} \mathrm{~cm}$ and this solution can easily be ruled out.

\section{GENERAL INVERSE COMPTON MODEL}

So far we have considered an SSC solution. However, it is interesting to note that Equations (4)-(7) apply when the prompt $\gamma$-rays are the IC scattering of low-energy external photons, provided that there is no significant relativistic bulk motion between the source of the seed photons and the IC electrons. For the UV solution, $\gamma_{e} \sim 50$ implies that the total kinetic energy should be much larger than the energy of the prompt $\gamma$-rays as the total internal energy of the electrons is much less than the rest-mass energy of protons. This leads to a severe energy budget problem. For the IR solution $(\alpha=-2.6)$ the Lorentz factor of the electrons is $\gamma_{e} \sim 2.5 \times 10^{3} Y^{-0.31}$; then the peak of the lowenergy emission is at $v_{L}=2.5 \times 10^{13} Y^{0.625}$ and the required peak flux density (not necessarily synchrotron emission) is $F_{v_{L}}=8.6 \times 10^{-19} Y^{-1.625} \mathrm{erg} \mathrm{cm}^{-2} \mathrm{~Hz}^{-1} \mathrm{~s}^{-1}$. Given the very small $v_{L}$, one can hardly satisfy $F_{v_{L}} \leqslant F_{\mathrm{BB}}\left(v_{L}\right)$ (or even stronger $\left.F_{v_{L} / 60} \leqslant F_{\mathrm{BB}}\left(v_{L} / 60\right)\right)$ for reasonable parameters.

\section{TWO ELECTRON POPULATIONS}

We now consider an alternative model in which the optical and soft $\gamma$-rays arise from synchrotron emission from the same physical region but from different populations of electrons. We denote these populations with subscripts $L$ and $\gamma$ for the lowerenergy band and the higher one, respectively. $\gamma$ and $N$ are the typical Lorentz factor and the total number of electrons, respectively, of each kind. As we assume a single emitting region, the magnetic field $B$ and the bulk Lorentz factor $\Gamma$ should be the same.

Using the peak synchrotron frequency relation $v_{p} \propto \gamma^{2} \Gamma B$ and the peak flux density $F_{v, p} \propto N \Gamma B$ :

$$
\frac{\gamma_{L}^{2}}{\gamma_{\gamma}^{2}}=\frac{v_{L}}{v_{\gamma}}
$$

and

$$
\frac{N_{L}}{N_{\gamma}}=\frac{F_{v, L}}{F_{\nu, \gamma}} .
$$

The total isotropic internal energy should be $\propto N_{e} \gamma$. Combining the above two equations, we obtain

$$
\frac{\gamma_{L} N_{L}}{\gamma_{\gamma} N_{\gamma}}=\frac{v_{L}^{1 / 2} F_{\nu, L}}{v_{\gamma}^{1 / 2} F_{\nu, \gamma}}=\frac{v_{\mathrm{opt}}^{1 / 2} F_{\nu, \mathrm{opt}}}{v_{\gamma}^{1 / 2} F_{\nu, \gamma}}\left(\frac{v_{L}}{v_{\mathrm{opt}}}\right)^{1 / 2+\tilde{\alpha}},
$$

where $\tilde{\alpha}$ is the spectral slope in the range $\left[v_{\mathrm{opt}}, v_{L}\right]$. If $v_{L}>v_{\mathrm{opt}}$, then clearly $\left(v_{L} / v_{\text {opt }}\right)^{1 / 2+\tilde{\alpha}}>1$. On the other hand, if $v_{L}<v_{\text {opt }}$, 
$\tilde{\alpha}<-1$ and again $\left(v_{L} / v_{\text {opt }}\right)^{1 / 2+\tilde{\alpha}}>1$. Using the observed values we obtain

$$
\frac{\gamma_{L} N_{L}}{\gamma_{\gamma} N_{\gamma}} \geqslant \frac{v_{\mathrm{opt}}^{1 / 2} F_{\nu, \mathrm{opt}}}{v_{H}^{1 / 2} F_{\nu, \gamma}} \sim 4 .
$$

Thus a peculiar condition of this model is that the energy of the lower-energy electron population that is responsible for producing the (relatively weak) optical signal exceeds that of the component producing $\gamma$-rays. Once more we are faced with an energy budget problem that makes the two synchrotron components model quite unlikely (see also R. Mochkovitch 2009 , in preparation).

\section{CONCLUSIONS AND DISCUSSIONS}

Piran et al. (2009) have recently shown that typical GRBs with normal (weaker than 12th magnitude) prompt optical emission cannot be produced by SSC of a softer component. The unique burst GRB 080319B had a very luminous prompt optical emission and one could expect, at first glance, that the prompt $\gamma$-rays were produced in SSC of the optical prompt emission. This was the accepted interpretation in the discovery paper (Racusin et al. 2008b) as well as in several others (Kumar \& Panaitescu 2009; Fan \& Piran 2008). The numerous detailed observations of this burst led to the hope that one can determine the physical parameters within the emitting regions here. However, a careful analysis reveals a drastically different picture. There is no reasonable SSC solution. One can make an even stronger statement and argue that it is unlikely that the prompt $\gamma$-rays are produced by inverse Compton scattering of seed photons produced by a source with no relativistic bulk motion relative to the IC electrons (regardless of the origin of the seed photons).

We also considered a situation with two populations of electrons that coexist in the same emitting region. Both populations emit synchrotron radiation with the less energetic electrons producing the optical while the more energetic ones produce the $\gamma$-rays. We have shown that even though the total energy released in the optical is orders of magnitude lower than the energy released in $\gamma$-rays; the population of lower-energy electrons should carry more energy-making, once more, the model energetically inefficient.

Combining these two results, we conclude that the optical emission and the soft $\gamma$-rays do not come from a single origin. This is the main conclusion of this Letter. Once we relax the condition that both modes of prompt emission are produced in the same region, there are many possibilities. However, even here the limits obtained in Section 2 indicate that the optical emission is produced at a very large radius which is most likely incompatible with internal shocks (note that typical internal shocks will have rather modest values of $\gamma_{e}$.). This raises the possibility that the optical emission is produced in this burst by the early external shock (possibly by the reverse shock). While there are several problems with this model (in particular the fast rise of the optical emission that is faster than expected in this case (Nakar \& Piran 2004)) they are less severe than those basic issues that arise with the internal shocks. The fact that the optical emission follows to some extent the $\gamma$-rays, but with a time delay of several seconds (C. Guidorzi 2008, private communication) is consistent with this model.

We thank P. Kumar, R. Mochkovitch, and Y. Fan for discussion. This work is supported by the Israel Science Foundation Center of Excellence in High Energy Astrophysics and by the Schwartzmann University Chair (T.P.), by a Marie Curie IRG and NASA ATP grant (R.S.), by the National Natural Science Foundation of China grant 10703002 and a Lady Davis Trust Post-Doctoral Fellowship (Y.C.Z.).

\section{REFERENCES}

Akerlof, C., et al. 1999, Nature, 398, 400

Blake, C. H., et al. 2005, Nature, 435, 181

Boër, M., et al. 2006, ApJ, 638, L71

Cwiok, M., et al. 2008, GCN Circ., 7445

Fan, Y. Z., \& Piran, T. 2008, Front. Phys. Chin., 3, 306

Golenetskii, S., et al. 2008, GCN Circ., 7482

Karpov, S., et al. 2008, GCN Circ., 7502

Katz, J. Z. 1994, ApJ, 432, L107

Klotz, A., et al. 2006, A\&A, 451, L39

Kobayashi, S., \& Sari, R. 2001, ApJ, 551, 934

Kumar, P., \& McMahon, E. 2008, MNRAS, 384, 33

Kumar, P., \& Panaitescu, A. 2009, MNRAS, 319, L19

Li, Z., \& Waxman, E. 2008, ApJ, 674, L65

Mészáros, P., \& Rees, M. J. 1999, MNRAS, 306, L39

Nakar, E., \& Piran, T. 2004, MNRAS, 353, 647

Piran, T. 2004, Rev. Mod. Phys., 76, 1143

Piran, T., Sari, R., \& Zou, Y. C. 2009, MNRAS, in press (arXiv:0807.3954)

Racusin, J. L., et al. 2008a, GCN Circ. 7427

Racusin, J. L., et al. 2008b, Nature, 455, 183

Sari, R., \& Piran, T. 1999a, ApJ, 520, 641

Sari, R., \& Piran, T. 1999b, ApJ, 517, L109

Vreeswijk, P. M., et al. 2008, GCN Circ. 7444

Wei, D. M., Yan, T., \& Fan, Y. Z. 2006, ApJ, 636, L69

Zou, Y. C., Dai, Z. G., \& Xu, D. 2006, ApJ, 646, 1098 